\title{
Les expériences migratoires différenciées d'étudiants français
}

De l'institutionnalisation des mobilités étudiantes à la circulation des élites professionnelles?

\section{Stéphanie Garneau}

\section{OpenEdition}

Journals

Édition électronique

URL : https://journals.openedition.org/remi/3731

DOI : $10.4000 /$ remi.3731

ISSN : $1777-5418$

Éditeur

Université de Poitiers

Édition imprimée

Date de publication : 30 juin 2007

Pagination : 139-161

ISBN : 978-2-911627-44-6

ISSN : 0765-0752

Référence électronique

Stéphanie Garneau, "Les expériences migratoires différenciées d'étudiants français », Revue européenne des migrations internationales [En ligne], vol. 23 - $n^{\circ} 1$ | 2007, mis en ligne le 30 juin 2010, consulté le 15 avril 2022. URL : http://journals.openedition.org/remi/3731 ; DOI : https://doi.org/ 10.4000/remi.3731

Ce document a été généré automatiquement le 15 avril 2022.

(C) Université de Poitiers 


\title{
Les expériences migratoires différenciées d'étudiants français
}

\author{
De l'institutionnalisation des mobilités étudiantes à la circulation des \\ élites professionnelles?
}

\author{
Stéphanie Garneau
}

Nous remercions le Conseil de recherche en sciences humaines du Canada (CRSH), le Fonds québécois de recherche Culture et Société (FQRSC), l'ARUC «Insertion des jeunes en région » et l'INJEP qui ont contribué financièrement à différentes étapes de cette recherche. De chaleureux remerciements vont également à Alexandra Gilbert dont les commentaires sur une première version de ce texte ont permis de lui apporter des modifications substantielles.

1 Au cours des dernières décennies, le nombre d'étudiants étrangers dans le monde s'est accru sous les effets conjugués de la globalisation, des grands processus de construction régionale et de l'évolution des politiques d'immigration. Les étudiants inscrits dans un établissement d'enseignement supérieur à l'extérieur de leur pays d'origine sont en effet passés de 240000 en 1960 à 1600000 en 2002, ce qui correspond à un rythme d'augmentation annuelle de 7 \% (Coulon et Paivandi, $2003: 4$ ).

2 Cette tendance observée à l'échelle mondiale est également constatée en France où à peine quelques 20000 étudiants étrangers étaient accueillis dans ses établissements d'enseignement supérieurs en 1960, alors qu'ils étaient 110800 en 1980 et 159500 en 2001/02 ${ }^{1}$. Simultanément à ces flux « entrants ", ce sont les déplacements des étudiants français vers les universités étrangères qui augmentent : alors qu'ils étaient 895 à partir étudier dans un autre pays européen en 1987, ce nombre est de 14821 dix ans plus tard, 19365 en 2002 (Commission européenne, 2004)².

3 Il ne s'agit pas ici d'affirmer que la migration pour études est un phénomène nouveau. La mobilité des étudiants et des professeurs, que ce soit sur une base individuelle ou lors du fractionnement des universités, était un trait caractéristique de la vie universitaire dès les XII ${ }^{e}$ et XIII ${ }^{e}$ siècles (Le Goff, 1960; Dedijer, 1968; Halary, 1994 ; Gaillard et Gaillard, 1999). De même, les premières décennies du XVII siècles ont connu l'apogée de la peregrinatio academica des enfants de la bourgeoisie, ce " "tour" plus ou moins complexe au cours duquel le jeune étudiant visitait successivement plusieurs universités 
et prenait ses grades sur le chemin du retour» (Charle et Verger, 1994: 44). Mais les migrations estudiantines résultent de l'action conjuguée de décisions politiques en matière d'éducation et d'immigration et de stratégies d'insertion économique et sociale de la part des individus et de leur famille; celles-ci varient en fonction des conjonctures politiques et économiques tant nationales qu'internationales. Aussi, partir à l'étranger pour étudier ne se fait plus aujourd'hui dans les mêmes conditions structurelles et idéologiques que par le passé, ce qui tend à transformer le paysage migratoire estudiantin.

4 En France, la période qui a suivi les indépendances de ses anciennes colonies fut celle de l'accueil des étudiants qui en provenaient. Les pouvoirs publics français faisaient alors appel à un discours d'aide au développement et de solidarité internationale afin de justifier les politiques, encore peu réglementées, d'accueil et de formation d'étudiants étrangers ${ }^{3}$. À cette époque, l'image des étudiants étrangers était positive et leur accueil en France s'inscrivait dans une logique universitaire (droit à l'éducation) et diplomatique (gestion des relations internationales) (Borgogno et Streiff-Fénart, 1996). Cette phase a été suivie, à partir du milieu des années soixante-dix, d'un resserrement des politiques d'immigration qui ne manqua pas d'affecter l'accueil des étudiants étrangers (Borgogno et Streiff-Fénart, 1996; Slama, 1999). Sous l'effet de la crise économique et d'une transformation qualitative des flux d'étudiants en provenance de l'étranger ${ }^{4}$, notamment, une double représentation des étudiants étrangers émerge alors : d'une part, on se met à les soupçonner d'être de faux étudiants venus en vérité travailler irrégulièrement en France; d'autre part, leur niveau de formation est jugé insuffisant et on leur reproche de porter atteinte à la renommée des établissements d'enseignement supérieur français. Diverses mesures restreignent de façon significative la sélection des candidats : obligation d'obtenir un visa de long séjour (sous condition d'une pré-inscription et de la preuve de ressources financières suffisantes), retour au pays au terme des études, non-renouvellement de la carte de séjour en cas d'échecs répétés aux examens, test linguistique obligatoire.

5 Aujourd'hui, alors qu'on se trouve dans un contexte à la fois de construction européenne, de mondialisation accélérée de l'économie et de contrôle accru de l'immigration, les politiques et les représentations de la mobilité étudiante internationale présentent de nouveaux traits. De fait, les gouvernements et les établissements d'enseignement supérieur français - comme d'ailleurs ceux d'autres sociétés occidentales - se sont lancés depuis une vingtaine d'années dans un vaste programme d'internationalisation de l'éducation et d'institutionnalisation de la mobilité internationale des étudiants. Les mesures entreprises ont des effets multiples qu'on commence à peine à distinguer et dont on évalue encore difficilement l'ampleur : multiplication des circulations étudiantes Nord-Nord dans le cadre d'accords interuniversitaires et intergouvernementaux fondés sur la réciprocité, accroissement des dispositifs de circulation et d'accueil destinés aux étudiants hautement spécialisés ( $2^{\mathrm{e}}$ et $3^{\mathrm{e}}$ cycles, stagiaires postdoctoraux) ou à ceux qui sont jugés solvables économiquement, resserrement des politiques d'accueil des étudiants en provenance du Sud (Borgogno et Streiff-Fénart, 1996; Slama, 1999; Latreche, 2001; Vaniscotte, Houguenague et West, 2003). Elles s'inscrivent, comme nous le verrons dans cet article, dans une mouvance néolibérale fondée sur les valeurs de compétitivité, de concurrence et d'excellence dans la perspective d'un "marché de la formation et de l'emploi hautement qualifié» (Borgogno et Streiff-Fénart, 1996 : 83). 
6 L'évolution des politiques et les transformations des flux d'étudiants étrangers questionnent l'égalité de l'accès à l'espace international de formation et, subséquemment, aux postes et secteurs d'emploi les plus qualifiés et compétitifs. En favorisant l'accès à des études à l'étranger à certaines catégories sociales, nationales et économiques d'étudiants, l'internationalisation de l'éducation et l'institutionnalisation accélérée de la mobilité académique ne contraignent-elles pas les autres à des formations locales parfois insuffisantes et inappropriées ou seulement moins distinctives? Par ailleurs, les dispositifs de mobilité académique mis de l'avant par les gouvernements et les établissements d'enseignement pour favoriser la migration "sortante " permettent-ils d'en généraliser l'accès à un plus large éventail de catégories sociales? Peut-on penser qu'ils facilitent effectivement leur insertion professionnelle ultérieure, voire qu'ils participent à la production de ces futures "élites" professionnelles identifiées dans d'autres recherches ${ }^{5}$, reléguant dans la foulée les «non mobiles » à des emplois subalternes?

7 Dans cet article, nous examinerons d'abord le cadre politique et institutionnel de la mobilité des étudiants en France et en Europe afin de mieux comprendre les conditions objectives dans lesquelles se déploient les circulations institutionnalisées des étudiants français ainsi que les représentations - plurielles - de la figure de l'étudiant étranger qui en découlent. À partir des expériences de jeunes Français ${ }^{6}$, à qui est donc destiné aujourd'hui un nombre accru de dispositifs institutionnels de mobilité étudiante internationale, nous nous intéresserons ensuite à la manière dont ceux-ci s'approprient l'espace international comme ressource dans leur processus de socialisation professionnelle. Par socialisation professionnelle, nous nous référons à l'ensemble des compétences et des connaissances acquises par l'individu dans une pluralité d'espaces d'activité formelle et informelle (école, travail, associations et autres lieux de sociabilité) et leur organisation progressive autour d'un engagement professionnel prééminent (Roulleau-Berger, 1991; 1995 ; 1999; Gauthier, 1997 ; Nicole-Drancourt et Roulleau-Berger, 2001). Bien que la socialisation professionnelle concerne aujourd'hui l'ensemble du cycle de vie des individus (Dubar, 1992), nous pouvons dire qu'il y a stabilisation en emploi lorsque cet engagement professionnel subjectivement (aspirations personnelles) et objectivement (qualifications, diplômes) revendiqué obtient la reconnaissance sur le marché du travail. Nous regarderons donc les conditions de construction du projet d'études à l'étranger, les ressources mobilisées afin de le mener à bien, les modes de transfert, sur les marchés du travail, des compétences et des savoirs acquis dans la mobilité, ainsi que les modalités de reconnaissance sociale, une fois de retour dans la société d'origine, des activités et des engagements réalisés à l'international.

8 Nous posons l'hypothèse que la globalisation de l'économie, l'internationalisation de l'éducation et l'institutionnalisation grandissante de la mobilité étudiante internationale se joignent aux stratégies d'insertion professionnelle et aux représentations subjectives des étudiants pour produire une valorisation différenciée des filières, des établissements et des espaces nationaux d'enseignement supérieur. Cette interaction entre les structures sociales et les acteurs migrants agit également à l'échelle nationale avec la hiérarchisation des parcours migratoires et des socialisations professionnelles des jeunes Français selon la rentabilisation des expériences internationales obtenue sur les marchés du marché travail. 


\section{Vers la réciprocité des accords et la mobilité des « élites »}

9 Le virage pris au cours des années soixante-dix, qui a consisté à resserrer les conditions d'entrée des étudiants en provenance des pays du Sud, a progressivement conduit les autorités publiques françaises à constater que leur échappait de la sorte un certain nombre de ces futures élites nationales qui se dirigent désormais vers les États-Unis et le Canada. La prise de conscience de l'existence d'un «marché de la formation supérieure [...] où s'affrontent les États-Unis avec 560000 étudiants étrangers, le Royaume-Uni avec 200000 étudiants étrangers, la France avec 130000 étudiants étrangers " (édufrance, 1998) amène la France a adopter à partir de la deuxième moitié de la décennie quatre-vingt-dix une attitude offensive en matière d'internationalisation de l'éducation. Elle crée notamment l'Agence édufrance dont la mission est de promouvoir l'offre éducative française à l'étranger auprès des étudiants de $2^{\mathrm{e}}$ et $3^{\mathrm{e}}$ cycles, de coordonner la coopération éducative internationale et d'améliorer les conditions d'accueil des étudiants étrangers en France.

10 Parallèlement à cette initiative nationale, le processus de construction européenne, qui concerne également l'éducation, se poursuit. Si par la création d'un espace européen de l'enseignement supérieur l'Europe aspire au renforcement de l'«appartenance à un espace social et culturel commun", elle cherche également à se positionner de façon compétitive sur le marché scientifique international :

" Partout, la vitalité et l'efficacité des civilisations se mesurent à l'aune de leur rayonnement culturel vers les autres pays. Nous devons faire en sorte que le système européen d'enseignement supérieur exerce dans le monde entier un attrait à la hauteur de ses extraordinaires traditions culturelles et scientifiques » (Déclaration de Bologne, 1999).

11 C'est face à cette concurrence mondiale perçue tant en France que dans la Communauté européenne que la préférence sera peu à peu accordée à l'accueil de deux catégories d'étudiants étrangers. La première est constituée des étudiants en provenance du Sud qui sont dans une spécialisation de $2^{\mathrm{e}}$ et de $3^{\mathrm{e}}$ cycles, futures élites qu'il ne faut pas perdre au profit de l'Amérique du Nord. Le programme Erasmus Mundus lancé en janvier 2004, qui offre à des étudiants des pays tiers une bourse d'excellence leur permettant de réaliser leur formation en Europe, est un exemple des plus récents de cette opération de (re)séduction menée auprès du fleuron des pays émergents. La seconde catégorie rassemble les étudiants « de la Communauté économique européenne et des grands pays à technologies avancées ", lesquels sont "solvables", ne souhaitent pas a priori rester, et avec lesquels la "réciprocité des avantages» est assurée ${ }^{7}$. Ce qui est primé, c'est la coopération avec les pays et les établissements d'enseignement jugés les plus profitables. Chaque étudiant en mobilité est d'ailleurs conçu par les acteurs institutionnels de la mobilité étudiante comme une forme d'ambassadeur qui participe autant que les représentants des services des relations internationales - officiellement chargés de conclure les accords de coopération interuniversitaire et d'échange d'étudiants - à l'attractivité de leur université d'attache à l'étranger (Vaniscotte, Houguenague et West, 2003 ; Breton et Lambert, 2003). La notion de réciprocité n'est pas secondaire puisque c'est la mobilité de "nos étudiants", à qui "nous devons [...] un système d'enseignement supérieur qui leur offre les meilleures chances de trouver leur propre domaine d'excellence", qui prendra une importance accrue tant dans les discours officiels que dans les politiques adoptées (Déclaration de la Sorbonne, 1998). Ce qui est 
de plus en plus mis de l'avant, c'est la nécessité de former la main-d'œuvre nationale aux nouvelles exigences de flexibilité et aux autres compétences dites internationales (plurilinguisme, carnet d'adresses internationales, maîtrise des codes culturels, etc.) (Wagner, 1998 ; Boltanski et Chiapello, 1999 ; Castells, 2001 ; Dezalay, 2004).

12 Ainsi, les pays membres de la Communauté mettent en place dès 1987 un programme du nom d'Erasmus ${ }^{8}$, lequel vise à promouvoir la mobilité intra européenne des étudiants et des enseignants. Entre 1987 et 2002, ce sont 1090560 étudiants européens qui effectuent un séjour universitaire à l'étranger dans ce cadre (Commission européenne, 2004). En 1999, cette initiative est encouragée par la Déclaration de Bologne qui entérine la création d'un espace européen de l'enseignement supérieur. Les pays signataires s'engagent alors à adopter une série de mesures telles que, notamment, un système de validation par crédits (ECTS) facilitant la reconnaissance des études faites à l'étranger, l'application de la réforme LMD (licence, master, doctorat) en vue d'harmoniser les diplômes européens, la composition d'un double cursus orientant soit vers le marché du travail, soit vers le master et le doctorat, et la promotion de la dimension européenne dans l'élaboration des différents programmes de l'enseignement supérieur (Déclaration de Bologne, 1999). Les régions, qui constituent également un acteur de plus en plus important de l'internationalisation de l'enseignement supérieur (Massit-Folléa et Épinette, 1992), ne sont pas en reste. La région Rhône-Alpes où s'est déroulée cette recherche est l'une des régions françaises qui encouragent le plus activement l'acquisition d'une formation à l'étranger9. En 1987, l'année même où démarre Erasmus, elle entre dans le mouvement de la mobilité étudiante en fondant le programme de Bourses régionales de formation à l'étranger (BRFE). Ces bourses, d'une valeur de $305 /$ mois pour un séjour pouvant s'étaler de quatre à huit mois, permettent à des étudiants rhônalpins de niveaux $\mathrm{Bac}+2 \mathrm{à} \mathrm{Bac}+5$ de poursuivre une partie de leur formation à l'étranger - à l'intérieur comme à l'extérieur de la Communauté européenne - tout en demeurant rattachés à leur université de départ, à laquelle ils continuent de payer leurs droits de scolarité. Elles ont donné une impulsion décisive à la mobilité étudiante internationale puisque le nombre de récipiendaires est en constante croissance depuis sa création : 1220 en 1990, 4861 en 2003 (Région Rhône-Alpes, 2004 : 6). On estime que grâce aux aides de la Région, près de $10 \%$ des étudiants rhônalpins expérimentent un séjour d'études à l'étranger ${ }^{10}$.

13 Ce rapide tour d'horizon nous éclaire sur les transformations qui touchent les discours officiels, les politiques et dispositifs institutionnels ainsi que les flux migratoires estudiantins en France au cours des années quatre-vingt-dix. Alors qu'en 1990, 56 \% des étudiants étrangers en France provenaient du Maghreb et des pays d'Afrique subsaharienne, cette proportion diminue à $49 \%$ en 2000. La proportion d'étudiants originaires d'un pays membre de la Communauté européenne passe quant à elle de $20 \%$ en 1990 à $29 \%$ en 2000 (Coulon et Paivandi, 2003 : 13). Simultanément, on assiste au glissement de la vocation idéologique ayant prévalu au lendemain de la Seconde Guerre mondiale, celle de la solidarité internationale, vers une instrumentalisation croissante du phénomène où les acteurs politiques recourent à un discours sur la préparation de la future main-d'œuvre aux défis des marchés du travail en transformation, tout en cherchant à positionner les marchés économique et éducatif nationaux dont ils sont les représentants sur la scène internationale. 


\section{La différenciation des expériences migratoires et des socialisations professionnelles}

14 Avec la multiplication des dispositifs de mobilité académique internationale destinés à la population universitaire française, d'autant plus prépondérante auprès des jeunes de la région Rhône-Alpes que nous avons rencontrés, de nouvelles structures d'opportunité se présentent à des catégories sociales d'étudiants plus diversifiées, ceux-ci pouvant progressivement construire leur carrière scolaire et professionnelle dans un espace géographique élargi ${ }^{11}$.

Les étudiants que nous avons interrogés ont sans conteste des origines sociales variées : dix ont un chef de famille qui appartient à la catégorie cadres et professions intellectuelles supérieures, treize à celle des professions intermédiaires, six à la catégorie artisans, commerçants ou chefs d'entreprise, trois à celle des employés et huit à la catégorie ouvriers. Mais cette relative généralisation de l'accès à un séjour d'études à l'étranger à des milieux sociaux différenciés conduit-elle aux mêmes résultats sur les marchés de l'emploi? Nous avons effectivement observé une différenciation de la rentabilité de l'expérience migratoire étudiante dans l'espace scolaire et professionnel qui n'est cependant pas automatiquement déterminée par la position initiale de l'individu dans la structure sociale. Les opportunités institutionnelles de mobilité juvénile se conjuguent aux ressources héritées du milieu social et familial d'origine pour produire l'accumulation d'un «capital spatial $»^{12}$ qui entre également dans la composition des expériences migratoires et des socialisations professionnelles. À la fois bagage de lieux (droits d'habiter, de travailler ou d'étudier, présence d'un réseau social, connaissances) et compétences à les gérer (capacité à se déplacer, à utiliser les lieux en économisant temps et énergie, à les agencer) (Lévy et Lussault, 2003), le capital spatial peut être échangé avec le capital social, culturel, économique ou symbolique (Bourdieu, 1979 ; 1980) en fonction des objectifs de l'acteur et des contextes (Roulleau-Berger et Shi, 2004).

Quatre types d'expérience migratoire et de socialisation professionnelle sont observés chez les étudiants en situation de mobilité selon le volume de capital spatial accumulé, le degré d'agencement à un projet professionnel des compétences acquises dans la mobilité, et les secteurs d'études et d'emploi. Il s'agit des expériences multispatiales intégrées, des expériences d'insertion professionnelle unispatiale, des expériences multispatiales aléatoires et des expériences unispatiales de précarité.

\section{Les expériences multispatiales intégrées}

17 Ce type de parcours migratoire est fondé sur une pluralité d'espaces sociétaux investis dans des cadres d'activité diversifiés. Il caractérise les jeunes qui sont partis dans plusieurs pays à plus d'une reprise pour réaliser des séjours successifs en colonie de vacances, en immersion linguistique, en coopération volontaire, en voyage, pour des études, pour un emploi ou pour un stage de formation. Chez ces individus, la production du capital spatial et l'accroissement de son volume prennent le plus souvent racine dans l'histoire familiale par le biais de pratiques concrètes ou virtuelles de l'espace. Dans le cas des pratiques concrètes, les familles expérimentaient une ou plusieurs expatriations dans le cadre du travail de l'un des parents, elles partaient fréquemment en vacances à l'étranger, elles retournaient régulièrement dans le pays 
d'origine des parents lorsque ceux-ci avaient émigré. Ces personnes possèdent donc déjà un capital spatial hérité de la famille qu'elles continueront à faire fructifier au cours de leur parcours ultérieur. Dans d'autres cas, les propensions à se déplacer et à séjourner à l'étranger proviennent de pratiques virtuelles de l'espace international, c'est-à-dire d'un rapport imaginaire et symbolique à l'ailleurs : des parents immigrés, un grand-père parti à la guerre, un oncle expatrié, une grand-tante "bohême", des parents « hippies » ayant sillonné les routes d'Europe, d'Asie ou d'Afrique pendant leur jeunesse, une éducation reçue dans une école bilingue ou internationale. «Le phénomène migratoire commence bien avant le déplacement physique, avec la prise de conscience d'un espace élargi " (Domenach et Picouet, 1995). Au même titre que les mobilités concrètes engendrent le développement de savoir-circuler (Tarrius, 2000), ces mobilités non actualisées provoquent une représentation élargie de l'espace d'action qui suscite des mobilités éventuelles.

La majeure partie des jeunes dont l'expérience migratoire répond au type multispatial intégré possède des volumes importants de capitaux initiaux (économiques, sociaux, culturels, symboliques, spatiaux) hérités de la position privilégiée de la famille dans la structure sociale. Néanmoins, certains d'entre eux qui sont originaires d'un milieu moins favorisé se sont retrouvés face à des structures d'opportunité qu'ils ont su mobiliser afin de partir à l'étranger, premier séjour qui interviendra dans le renforcement des autres capitaux et deviendra l'événement fondateur d'un parcours multispatial. Ici, l'école et son offre de voyages linguistiques et culturels, les comités d'entreprise qui permettent aux enfants d'ouvriers de partir en colonie de vacances à l'étranger, les organismes de soutien aux séjours linguistiques pendant la période estivale, jouent un rôle primordial puisqu'ils facilitent l'accès matériel - voire psychologique - à l'espace international à des jeunes originaires d'un milieu social où le capital économique est faible et où la mobilité ne fait pas partie des attentes d'occurrence normale.

19 Les aspirations à investir l'espace international dans le cadre des études ou de la profession éventuelle trouvent leur origine dans ces expériences préalables de mobilité internationale, lesquelles orientent plutôt précocement le choix des langues apprises au lycée, de la filière d'études universitaires et du pays privilégié pour la réalisation du séjour académique. Les aspirations professionnelles et la volonté de parcourir le monde se mêlent d'ailleurs souvent à une profonde quête identitaire qui assure une cohérence des activités et des choix de destination, comme dans le cas de cette jeune femme d'origine espagnole qui partira étudier en Espagne :

« Dans ma tête - ce qui est encore actuel - j'aimerais beaucoup y vivre. Donc c'était une manière à moi de me faire ma petite expérience parce que les gens autour de moi me disaient "ah, tu verras, tu sais l'été les choses sont différentes, c'est les vacances, etc. Tu verras si tu y vas et si tu travailles, les choses sont pas..." Et bon, je voulais vraiment voir la vie quotidienne et pas simplement la vie de vacances... " (Sabine, 25 ans, licence en Espagne).

Nombreux sont les jeunes Français issus de l'immigration qui se sont appropriés les dispositifs de mobilité étudiante dans le but d'approfondir leurs origines culturelles, de mieux maitriser leur langue maternelle et de définir un projet éventuel d'insertion professionnelle dans le pays de leurs parents. L'institutionnalisation de la mobilité des étudiants semblerait contribuer concrètement à la construction d'une relation non seulement identitaire mais aussi socio-économique avec le pays des origines (Hily et 
Oriol, 1993 ; Santelli, 1999) et ainsi faciliter le développement de liens transnationaux (Meintel, 1993).

Chez ces étudiants, les capitaux se renforcent mutuellement au fur et à mesure des séquences de mobilité internationale, des connaissances et compétences acquises et des obstacles et opportunités qui se présentent à eux. Ce qui ne signifie aucunement que leurs actions sont le fruit d'un calcul rationnel initial coûts/bénéfices. Leurs aspirations, leurs objectifs et leurs stratégies naissent et disparaissent, se modifient, s'ajustent et évoluent en fonction des conditions objectives et de leurs capacités à mobiliser les ressources de façon pertinente selon les contextes. Ils construisent leur parcours spatial, scolaire et professionnel de façon progressive, organisant le prochain projet de mobilité en fonction de leurs connaissances accumulées (sur une société particulière, une langue, un type d'activités professionnelles, etc.), de la densification et $\mathrm{du}$ déploiement de leur réseau par-delà les frontières nationales, de l'accès à de nouvelles structures de financement, etc.

Ayant préalablement fait des choix académiques qui sont d'une façon ou d'une autre orientés vers l'interculturel et l'international (études des langues, affaires internationales, géographie, etc.), et connaissant déjà les ressources auxquelles ils peuvent recourir afin de mener à bien leur projet, les dates limites pour le dépôt des dossiers ainsi que les pré-requis pour partir (connaissance suffisante de la langue, cours et nombre d'unités préalables), ces étudiants sont mieux à même d'entreprendre les démarches et de prévenir les obstacles éventuels. Les savoir-circuler, les "savoir-

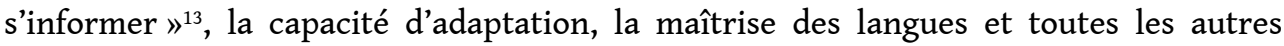
aptitudes qu'ils ont acquises au cours de leurs mobilités antérieures permettent une économie de temps, d'énergie et de problèmes qui facilite leur installation et leur adaptation aux sociétés d'accueil.

Aussi, cette organisation plus ou moins précoce de la carrière scolaire et professionnelle, qui donne à voir une certaine continuité des engagements divers entrepris dans une pluralité d'espaces sociétaux, une accumulation des connaissances et des compétences, un renforcement des capitaux, de même qu'un déroulement sans heurts majeurs du séjour d'études à l'étranger, facilite la reconnaissance des expériences au retour, tant de la part de l'établissement d'enseignement d'origine que du marché du travail. Contrairement à d'autres étudiants, comme nous le verrons plus loin, ces jeunes se trouvent dans des conditions institutionnelles où les séjours académiques sont fort bien organisés et disposent des ressources et des compétences personnelles qui leur permettent de remplir toutes les exigences du programme et d'obtenir les équivalences des cours effectués à l'étranger. De même, les possibilités institutionnelles de mobilité internationale et le renforcement réciproque de leurs capitaux semblent agir conjointement tout au long de leurs expériences pour faciliter la continuité des engagements et leur hiérarchisation progressive en faveur d'un rôle professionnel relativement précis lié à l'international. Ces individus connaissent par conséquent une transition de l'école à l'activité professionnelle relativement souple et rapide pour atteindre une position socioprofessionnelle et un statut qualifiés. Ils sont ingénieurs, universitaires, consultants et fonctionnaires à la Commission européenne, ils ont des contrats à durée indéterminée et ils sont appelés à se déplacer dans le cadre de leur emploi, à communiquer régulièrement avec des filières étrangères, à puiser quotidiennement dans un registre plus ou moins large de codes culturels et à faire usage de plusieurs langues. 


\section{Les expériences d'insertion professionnelle unispatiale}

24 de la majeure partie des engagements et des activités sur l'espace sociétal d'origine. Le séjour d'études est généralement la seule expérience internationale réalisée par l'étudiant sans sa famille. Lorsqu'il est parti à plus d'une reprise, c'est dans le même pays et pour y réaliser la même activité, soit des études. Les individus qui s'inscrivent dans ce type d'expérience migratoire de socialisation professionnelle disposent de volumes de capitaux économique, social et symbolique différenciés mais ils ont en commun de n'avoir pas hérité d'un fort volume de capital spatial. Dans les cas où le voyage faisait partie des pratiques familiales, ils n'ont pas poursuivi l'accumulation d'expériences spatiales au cours de l'adolescence. Ils ont préféré rassembler la majeure partie de leurs ressources et de leurs énergies dans le but de réussir leurs études, comme c'est le cas notamment des élèves qui ont fait les classes préparatoires. Néanmoins, plongés dans des conditions institutionnelles où la mobilité étudiante internationale est bien organisée et financée, la possibilité de réaliser une partie de la formation dans une université étrangère est venue à la rencontre de leurs intérêts. Ces personnes sont mues, pour des raisons liées à leur histoire personnelle et familiale, à la forte compétitivité dans leur filière de formation ou au faible degré d'ouverture du marché du travail dans leur secteur professionnel, par une profonde incertitude quant à leur avenir et à « leur place » dans la société. Elles s'orientent généralement vers des domaines professionnels "nationaux " tels que l'enseignement et le journalisme et n'abordent pas tant le séjour d'études comme une entrée éventuelle dans une carrière professionnelle internationale que comme une expérience pouvant les distinguer de leurs confrères quand le temps sera venu de soumettre leur candidature à un poste.

Ici, les différents volumes de capitaux hérités et acquis de l'individu, plutôt que d'agir les uns sur les autres et de se consolider mutuellement, comme dans les socialisations précédentes, tendent à s'unir et à se concentrer pour le renforcement du capital culturel et symbolique. En d'autres termes, le répertoire de ressources est moins mobilisé en vue d'accroître un patrimoine de lieux et des compétences spatiales qu'en vue d'élever le niveau de formation et de spécialisation professionnelle. La majeure partie des actions est posée en fonction de l'entrée prochaine sur le marché du travail. Le volume de capital spatial, après une augmentation due à une première appropriation de l'espace international, tend à se stabiliser et à demeurer constant. De plus, contrairement aux jeunes précédents qui font leur entrée dans l'enseignement postsecondaire en ayant en tête un projet relativement bien élaboré d'études à l'international, ceux dont nous parlons ici ne connaissent pas déjà cette possibilité et moduleront leurs objectifs en fonction, d'une part, de l'offre institutionnelle (établissements d'enseignement avec lesquels des ententes sont signées, durée des séjours proposés, supplément aux bourses pour certaines destinations) et, d'autre part, de leurs ressources personnelles, notamment leur maîtrise des langues étrangères. Les cas ne sont pas rares où des étudiants souhaitaient partir dans une université américaine pour le prestige qui s'y rattache et pour approfondir leur connaissance de l'anglais mais qui se sont finalement vus redirigés vers un autre pays en raison de la rareté des accords interuniversitaires conclus avec les États-Unis ou de leurs faibles aptitudes dans la langue. 
«Au début je voulais partir dans une université américaine anglophone. C'était un défi que je m'étais lancé, faire un écrit en anglais. Et puis donc j'ai passé le TOEFFLE mais j'ai eu un nombre de points insuffisant pour entrer dans une université américaine » (Stéphane, 27 ans, maîtrise au Québec).

À l'instar de ce jeune homme, qui prendra finalement la direction du Québec, les étudiants acceptent néanmoins de modifier leurs aspirations initiales, misant plutôt sur les bienfaits potentiels de toute expérience à l'étranger, quelle que soit la destination.

Ces individus reviennent dans leur société d'origine au terme de leur expérience afin d'y compléter leur formation universitaire ou d'y entreprendre des démarches d'insertion en emploi. La plupart du temps, comme c'est le cas des étudiants qui comptent se présenter au Certificat d'aptitude au professorat de l'enseignement secondaire (CAPES), cet itinéraire était prévu. Les autres, à la suite d'une rencontre amoureuse ou d'une expérience positive, ont pensé pendant un moment prolonger leur séjour à l'étranger. Ils sont néanmoins rentrés en France, tantôt par crainte que le diplôme étranger ne soit pas reconnu, tantôt parce qu'en l'absence de supports institutionnels financiers, ils ne disposaient pas des moyens économiques nécessaires.

Dans ce type d'expérience, la transition au monde du travail se fait de manière différenciée selon les domaines professionnels. Quoi qu'il en soit, les expériences de travail s'enchaînent et s'accumulent de façon continue et plus ou moins cohérente jusqu'à l'obtention d'un poste stable. Certains jeunes Français qui se dirigent vers l'enseignement ont vécu la préparation du CAPES péniblement et admettent que le séjour d'études l'année précédente n'a pas été étranger à leur mauvaise expérience.

«Le CAPES d'histoire! Et ça a été horrible, ça a été une année très difficile. Parce que sortir

d'une année, d'une année insouciante, en fait, peut-être la seule année un peu insouciante et

de me replonger dans quelque chose de très scolaire avec beaucoup de stress... Ça n'a vraiment pas été facile. Surtout, me remettre au boulot, quoi » (Anne-Marie, 28 ans, licence en Allemagne).

Parmi les quatre enquêtés inscrits au CAPES à leur retour de l'étranger, deux s'y sont repris à trois fois avant de le réussir et les deux autres ont abandonné leurs aspirations à devenir enseignant au bout de deux échecs. Tous vivent cette période comme une épreuve. D'autres jeunes cumulent des stages et des contrats à durée déterminée pendant quelques mois, mais ces expériences s'ordonnent autour d'un engagement professionnel relativement bien défini. Les connaissances et les compétences acquises présentent une certaine continuité et finissent par obtenir une sanction positive sur les marchés du travail. Dans certains cas, la transition se fait progressivement, du stage de formation au poste permanent, en passant par le contrat à durée déterminée. Dans les domaines où la conjoncture économique offre des débouchés professionnels et pour ceux des enquêtés qui possèdent des diplômes de troisième cycle, les démarches de recherche d'emploi paraissent si bien aller de soi que certains sont embauchés avant même la fin de leur période de formation.

Les individus ici représentés ont en commun que leurs différents engagements sociaux et leur usage de l'espace international s'agrègent pour offrir un parcours objectivement cohérent au regard d'un métier ou d'une profession qui finit par obtenir la reconnaissance des employeurs. S'ils ne sont pas toujours en mesure d'affirmer avec certitude qu'ils ont été retenus lors d'un concours d'entrée ou d'un entretien d'embauche en raison même de leur séjour de formation à l'étranger, ils disent néanmoins avoir été longuement questionnés à ce sujet dans toutes les situations où cela s'avérait pertinent. 


\section{Les expériences multispatiales aléatoires}

31 Les expériences aléatoires se caractérisent par un système spatial composé de plusieurs espaces cumulés dans le temps. À l'instar des expériences multispatiales intégrées, les itinéraires sont ponctués de nombreuses phases de mobilité dans des lieux divers et sous des formes diversifiées. Mais, contrairement à leurs prédécesseurs, les étudiants mobiles dont la socialisation professionnelle présente un aspect aléatoire cumulent du capital spatial sans que celui-ci ne vienne grossir d'autres capitaux. Si, grâce à leurs séjours linguistiques, leurs voyages d'aventure et leur formation à l'étranger, ils parlent plusieurs langues et développent des capacités d'adaptation et des savoir-circuler, ces compétences spatiales ne s'unissent pas autour d'un projet académique ou professionnel défini. Les ressources apparaissent dispersées, les rôles multiples et les séquences de mobilité internationale discontinues.

Deux cas de figure, l'un vécu sur un mode positif et l'autre sur un mode négatif, peuvent illustrer les expériences de type aléatoire. Dans le premier, les acteurs ont le désir affiché, conscient et revendiqué de différer leurs engagements professionnels et de s'inscrire temporairement dans un mode de vie caractérisé par un certain nomadisme. Quelques-uns d'entre eux ont le loisir de se consacrer à leurs intérêts multiples grâce à la position socioprofessionnelle privilégiée de leurs parents. D'autres, moins favorisés, optent délibérément pour des formes d'emploi qui ne les enferment pas localement et choisissent de ne pas s'attacher aux choses matérielles, cela afin d'être en mesure de voyager. Ce type d'expérience où les engagements sociaux sont nombreux et se dispersent dans un espace international aux lieux multiples est généralement voulu temporaire, pour une période de trois à cinq ans. Les individus correspondants, qui savent gérer l'incertitude, laissent place à l'imprévu.

Dans le second cas de figure rencontré, les individus sont en position de tension, voire de fragilité. Ils vivent des complications sentimentales, ils connaissent des conflits familiaux, ils subissent des accidents biographiques, telle la maladie, qui interfèrent avec leurs intentions professionnelles initiales, non sans provoquer une distension entre les aspirations personnelles et des assignations à des rôles qu'ils refusent. Parfois, c'est la dispersion même des activités spatialisées et des expériences interculturelles qui nourrit la confusion chez des individus déjà en proie à des questionnements existentiels et à l'incertitude quant à leur avenir professionnel et à leur futur rôle dans la société. Lorsqu'en plus, au fil de leurs pérégrinations, ils se sont détachés peu à peu de leurs engagements dans la société d'origine, ils vivent leurs retours dans la douleur : ils se sentent seuls et isolés dans un environnement social et culturel qui est pourtant le leur. Leurs difficultés tendent alors à être associées à la société d'origine tandis que l'étranger est fantasmé. Aussi le capital spatial peut-il se présenter comme une contrainte lorsqu'il mène à l'inanition du réseau social dans la société d'origine et qu'il fait de tout départ une solution. La multiplication précipitée et désordonnée des séjours à l'étranger, lorsque ceux-ci ne tendent pas vers la conversion des savoirs personnels en compétences professionnelles, mène en effet à la dispersion des ressources et à une sorte de fuite en avant.

Ces individus enchaînent les emplois précaires sans que ceux-ci ne semblent vouloir conduire à l'obtention d'un poste répondant à leurs aspirations. Ils n'hésitent pas à exprimer leur déception à l'égard de ce qu'ils ressentent comme une dénégation de 
leurs qualifications et des expériences acquises à l'étranger. Ils ont l'impression que les acteurs institutionnels (employeur, ANPE, etc.) avec lesquels ils entrent en interaction lors de leurs recherches d'emploi les suspectent d'être instables ou, lorsqu'ils sont sur le marché du travail, que leurs expériences antérieures sont dénigrées : «Ils sont du style à me dire "ici c'est pas l'Australie". Ou "Lyon, c'est pas Sydney" " (Florence, 27 ans, maîtrise en Australie). Ce manque de reconnaissance rencontré dans le monde du travail semble dépendre des caractéristiques du secteur d'emploi (à vocation plutôt locale, qui ne nécessite pas des compétences internationales) ou du contexte situationnel (employeur peu ouvert sur l'étranger, collègues manifestant un certain ethnocentrisme, etc.). Ces enquêtés ne sont pas satisfaits de leur situation professionnelle et ont comme projet d'en changer ou de repartir à l'étranger.

\section{Les expériences unispatiales de précarité}

Enfin, d'autres individus développent un parcours où la majeure partie des engagements sociaux sont pris dans la société d'origine sans toutefois mener à une accumulation progressive de compétences liées à un rôle professionnel relativement circonscrit. Si aucun des enquêtés rencontrés ne se trouve dans une situation de vulnérabilité extrême, ils ont cessé leurs études sans avoir obtenu le diplôme, ils occupent un emploi à statut précaire dans un domaine - souvent dans le secteur des services - qui n'est pas lié à leur champ d'études, ils sont faiblement rémunérés ou sont au chômage. Les compétences acquises à l'école, dans les emplois occupés et dans les activités hors travail ne sont pas transférées dans des activités professionnelles. Les projets d'avenir sont imprécis et ne sont pas en cours d'élaboration.

Tout comme les autres membres de leur famille, ces jeunes n'ont pas à leur actif un patrimoine de lieux inter-reliés par un réseau social décloisonné et multipolarisé. Ils n'ont préalablement pas développé de capacités et d'aptitudes à se déplacer dans un espace international. Le séjour d'études à l'étranger est l'unique activité internationale qu'ils ont expérimentée. Les rôles d'expatrié ou de voyageur ne faisaient tout simplement pas partie de leur répertoire de rôles envisageables. Ce sont les conditions institutionnelles favorables - promotion intensive, voire personnalisée, de la mobilité étudiante au sein de la faculté, disponibilité de supports financiers - qui sont venues ouvrir une brèche dans l'inventaire des possibles. Pour certains, c'est d'ailleurs l'accès à un financement qui a facilité l'obtention de l'appui - au moins moral - des parents lorsque le budget familial est serré ou que les dépenses nécessitées par un séjour à l'étranger sont jugées inutiles: "Mon père un peu plus froid, au départ, niveau question financière. Bon après, la bourse, ça a quand même bien aidé" (Juliette, 27 ans, maîtrise au Québec). Ces étudiants n'ayant pas déjà en tête un projet bien formulé d'études à l'étranger et leurs connaissances linguistiques se limitant pour la plupart à leur langue maternelle, ils organisent leur séjour en fonction des pays dont ils parlent la langue et des établissements étrangers avec lesquels leur université d'attache possède une entente. Étant donné la communauté de langue et l'ancienneté de la coopération universitaire franco-québécoise, nombre d'entre eux se sont dirigés vers le Québec.

37 Ces acteurs sociaux se retrouvent en situation de précarité sur le marché du travail. Ils enchaînent les périodes d'activité et de chômage, les petits boulots et les emplois instables. Pour certains, encombrés de problèmes personnels (désordres amoureux, conflits familiaux, angoisse quant à l'avenir, etc.), cette situation d'instabilité est 
occasionnée par une difficulté personnelle à transiter d'un espace socioculturel à l'autre, notamment lors du retour dans la société d'origine. À l'instar des jeunes qui vivent des expériences migratoires aléatoires, l'ailleurs est fantasmé et tous les efforts sont alors concentrés autour de l'idée de repartir dans la société qui les a accueillis. Cette sur-idéalisation de l'expérience passée se joint au manque de ressources pour freiner la mise en route de projets (de formation, de carrière...) tant dans la société d'origine qu'à l'étranger.

Cette nostalgie est d'autant plus paralysante que l'expérience de mobilité internationale conduit à la perte de ressources économiques et culturelles. Des jeunes se sont endettés financièrement au cours de leur séjour. À leur retour ils ont été forcés, faute d'un capital social ou économique nécessaire, de négliger ou d'abandonner leurs études afin d'occuper des emplois précaires, des « petits boulots » qui ne favorisent pas l'accumulation d'expériences professionnelles valorisées. D'autres ont échoué des cours effectués à l'étranger ou se sont heurtés au refus de leur université d'origine de reconnaître les unités obtenues dans l'établissement d'accueil. Il s'agit souvent de cas où l'étudiant, à son arrivée dans l'établissement d'accueil, constate que les cours préalablement choisis avec son responsable de programme ne sont finalement pas offerts. La vitesse avec laquelle il doit faire d'autres choix et les difficultés de communication avec son université d'origine conduisent, au retour, à de mauvaises surprises. Plutôt que d'accroître le volume de capital culturel, l'expérience des études à l'étranger peut provoquer l'effet inverse lorsqu'elle mène à la sortie prématurée du monde universitaire.Dans les expériences unispatiales précaires, les remises en question de certains choix scolaires et professionnels, les événements de la vie personnelle et l'accumulation d'épreuves négatives contraignent les jeunes à emprunter des directions qu'ils n'ont pas choisies, comme dans le cas de cette étudiante en histoire qui échoue à trois reprises le CAPES ou de cette autre qui occupe un poste de salarié sans lien avec son domaine d'études.

En somme, les acteurs sociaux dont l'expérience migratoire est unispatiale et précaire rencontrent des difficultés à faire valoir les qualifications acquises à l'université ainsi qu'à travers l'expérience de mobilité étudiante internationale. Un domaine d'études qui n'est pas lié à l'international, de faibles débouchés professionnels dans leur domaine de formation, des complications administratives relatives à la faiblesse de l'encadrement de leur échange universitaire, un faible volume de capital spatial qui ne leur permet pas de parer à ce type d'obstacles, l'enchaînement d'épreuves personnelles... l'ensemble de ces facteurs structurels, institutionnels et personnels s'entremêle pour prévenir l'organisation des activités autour d'un engagement professionnel et la rentabilisation du séjour à l'étranger sur le marché du travail.

Par l'identification de ces quatre expériences migratoires de socialisation professionnelle, il n'est nullement question d'essentialiser le vécu des étudiants et encore moins de les catégoriser (Schnapper, 1999). Aucune des expériences ici décrites ne se retrouve telle quelle à l'état pur. Selon la tranche du parcours considérée, on peut dire que le vécu des personnes que nous avons rencontrées se rapproche de plusieurs types à la fois. Ces quatre expériences sont des types idéaux au sens de Weber. Elles doivent être appréhendées comme un «tableau de pensée homogène » (Weber, 1965 : 181), une grille de lecture qui nous permet de mieux comprendre comment des individus qui se trouvent dans des conditions économiques et politiques d'internationalisation des marchés du travail et de l'enseignement supérieur s'approprient l'espace international 
au cours de leur parcours de socialisation professionnelle en fonction de leurs ressources personnelles et familiales initiales.

\section{Conclusion}

Analyser les expériences migratoires et les processus de socialisation professionnelle de jeunes Français à la lumière des dispositifs et des représentations politiques de la mobilité internationale des étudiants en France et en Europe permet de révéler la production de certaines formes d'inégalités à plusieurs niveaux et échelles spatiales. Les politiques réglementant l'accueil des étudiants étrangers et les dispositifs institutionnels favorisant certains flux migratoires estudiantins à l'échelle régionale, nationale et/ou européenne ${ }^{14}$ semblent peu à peu conduire à une hiérarchisation des circulations étudiantes et, par conséquent, des espaces éducatifs nationaux ${ }^{15}$. En privilégiant la réciprocité des avantages garantie par les ententes de coopération scientifique et d'échange d'étudiants, ce sont les étudiants en provenance des pays industrialisés, sinon les étudiants de haut niveau qui sont jugés être les futures élites de leur pays et ceux qui sont solvables et rentables, c'est-à-dire qui financent eux-mêmes leurs études, qui ont la priorité. Un clivage apparaît ainsi progressivement entre les étudiants internationaux en provenance des pays occidentaux qui circulent sur les voies pavées des accords de réciprocité conclus entre les gouvernements et les établissements d'enseignement (les couches privilégiées du Sud y étant ici associées) et les étudiants étrangers de classe moyenne en provenance de pays plus démunis qui cherchent, en dépit de politiques d'accueil contraignantes, à se frayer un chemin vers les universités occidentales.

Les étudiants français que nous avons rencontrés font partie de ce public récemment ciblé de façon intensive par les politiques d'internationalisation de la formation. Or, nous avons vu qu'il s'opère également parmi eux une diversification des situations selon le degré de rentabilité des expériences migratoires sur les marchés du travail. Les quatre types de socialisation professionnelle que nous avons présentés témoignent en effet d'un continuum hiérarchisé des expériences migratoires d'étudiants provenant d'un même espace régional, où certains connaissent une insertion dans l'emploi plus réussie que d'autres. Les origines sociales et les programmes de mobilité internationale destinés à la jeunesse agissent ensemble sur l'accumulation du capital spatial pour intervenir de façon différenciée sur le choix de la filière d'études, le travail d'ordonnancement de leurs compétences autour d'un projet défini de carrière et la reconnaissance subséquente de leurs expériences dans des secteurs d'emploi inégalement compétitifs et valorisés en période de mondialisation économique. À moins d'un accès à d'importants dispositifs organisationnels et financiers de mobilité juvénile en amont mais aussi en aval du séjour d'études, qui permet aux jeunes issus des milieux les moins privilégiés socialement, culturellement et économiquement d'accumuler un capital spatial de façon précoce et dans la durée, ceux qui proviennent d'un milieu familial favorisé ont une longueur d'avance dans l'appropriation et l'usage de l'espace international au cours de leur socialisation professionnelle.

Les discours des autorités politiques et éducatives participent à la propagation de l'idéologie aujourd'hui en vigueur dans le monde de l'entreprise où la mobilité, la flexibilité, l'adaptabilité et la capacité à créer et entretenir un réseau délocalisé constituent les principes au nom desquels se distinguent de plus en plus les « grands » 
des «petits» (Boltanski et Chiapello, 1999). Mais tous les étudiants, étant donné leurs origines sociales, sociétales et disciplinaires, n'ont pas la même égalité d'accès à l'espace international ni les mêmes capacités à démêler une offre éducative (de programmes, de diplômes, etc.) aussi transnationale que floue et incertaine, du moins en l'état actuel, notamment en termes de reconnaissance ultérieure des titres sur les marchés nationaux de l'emploi. Cela est d'autant plus vrai et discrétionnaire lorsque dans le même temps, des mesures politiques sont mises en place pour contrer l'accès à des études à l'étranger à d'autres catégories nationales de population étudiante.

Aussi, il se peut que l'institutionnalisation de la mobilité internationale de certaines catégories d'étudiants, qui tend aujourd'hui à obéir davantage à une logique mercantile et instrumentale qu'à une logique universitaire, se joigne aux stratégies d'appropriation subjective et matérielle de l'espace international par les étudiants pour donner lieu à un jeu de "classement, déclassement et reclassement ${ }^{16}$ des espaces éducatifs nationaux et à la production subséquente de mobilités académiques «en cascade ». Dans cette optique, les étudiants les moins bien positionnés dans la structure sociale et économique internationale seraient contraints à une formation locale, ceux qui se trouvent dans une position intermédiaire pourraient moduler leur cursus académique en fonction des ententes interuniversitaires et intergouvernementales dont dispose leur établissement d'origine, et ceux qui sont les mieux pourvus, en un effort ultime de distinction, auraient le loisir de se diriger vers les universités les plus prestigieuses et les programmes de formation les mieux adaptés à leurs aspirations professionnelles.

\section{BIBLIOGRAPHIE}

ARWU (2005) Academic Ranking of World Universities, Jiao Tong University, Shanghai, http:// ed.sjtu.cn/rank/file/ARWU-M\&P.pdf, dernière consultation le 3 mai 2006.

BOLTANSKI, Luc et Ève CHIAPELLO (1999) Le nouvel esprit du capitalisme, Paris, Gallimard, 843 p.

BORGOGNO, Victor et Jocelyne STREIFF-FÉNART (1996) L'accueil des étudiants étrangers en France : évolution des politiques et des représentations, Cahiers de l'URMIS, n² 2-3, pp. 73-88.

BOURDIEU, Pierre (1978) Classement, déclassement, reclassement, Actes de la recherche en sciences sociales, $\mathrm{n}^{\circ} 24$, novembre, pp. 2-22.

BOURDIEU, Pierre (1979) Les trois états du capital culturel, Actes de la recherche en sciences sociales, vol. 30, pp. 3-6.

BOURDIEU, Pierre (1980) Le capital social, notes provisoires, Actes de la recherche en sciences sociales, vol. 31, pp. 2-3.

BRETON, Gilles et Michel LAMBERT Éds. (2003) Globalisation et universités. Nouvel espace, nouveaux acteurs, Québec, Les Presses de l'Université Laval, 264 p.

CASTELLS, Manuel (2001) La société en réseaux, «L'ère de l'information », tome I Paris, Fayard, $671 \mathrm{p}$. 
CHARLE, Christophe et Jacques VERGER (1994) Histoire des universités, Paris, PUF, $126 \mathrm{p}$.

COMMISSION EUROPÉENNE (2004) Socrates - Erasmus. Le programme de la Commission européenne dans le domaine de l'enseignement supérieur,

http://www.europa.eu.int/comm/education/programmes/socrates/erasmus/stat en.html, dernière consultation le 23 octobre 2005.

COULON, Alain et Saeed PAIVANDI (2003) Les étudiants étrangers en France : l'état des savoirs, rapport pour l'Observatoire national de la vie étudiante, Paris, mars, $41 \mathrm{p}$.

DÉCLARATION DE BOLOGNE (1999) L'espace européen de l'enseignement supérieur, déclaration commune des Ministres européens de l'Éducation réunis à Bologne le 19 juin.

DÉCLARATION DE LA SORBONNE (1998) Harmoniser l'architecture du système européen d'enseignement supérieur, à l'occasion du $800^{\mathrm{e}}$ anniversaire de l'Université de Paris, déclaration conjointe des quatre ministres en charge de l'enseignement supérieur en Allemagne, en France, en Italie et au Royaume-Uni, Paris, en Sorbonne, le 25 mai.

DEDIJER, Stevan (1968) “Early” Migration, in Walter Adams, The Brain Drain, New York, Macmillan, pp. 9-28.

DEZALAY, Yves (2004) Les courtiers de l'international. Héritiers cosmopolites, mercenaires de l'impérialisme et missionnaires de l'universel, Actes de la recherche en sciences sociales, $\mathrm{n}^{\circ} 151-152$, pp. 5-35.

DOMENACH Hervé et Michel PICOUET (1995) Les migrations, Paris, PUF, 127 p.

DUBAR, Claude (1992) Formes identitaires et socialisation professionnelle, Revue française de sociologie, XXXIII, pp. 505-529.

ÉDUFRANCE (1998) Fiche de présentation d'Édufrance, www.st-andrews.ac.uk/ filtafr/texte1.htm, dernière consultation le 8 mai 2006.

FINDLAY, Allan M. et al. (1999) Foreign academics in a global city: the case of Hong Kong, Revue européenne des migrations internationales, vol. 15, no 1, pp. 121-137.

GAILLARD, Anne Marie et Jacques GAILLARD (1999) Les enjeux des migrations scientifiques internationales. De la quête du savoir à la circulation des compétences, Paris, L'Harmattan, 233 p.

GARNEAU, Stéphanie (2006) Les mobilités internationales à l'ère de la globalisation. Une comparaison sociologique des carrières spatiales et des socialisations professionnelles d'étudiants français et québécois, thèse de doctorat sous la direction de Laurence Roulleau-Berger, Faculté d'anthropologie et de sociologie, Université Lumière-Lyon 2, 437 p.

GAUTHIER, Madeleine (1997) La migration et le passage à la vie adulte des jeunes d'aujourd'hui, in Madeleine Gauthier Éd., Pourquoi partir ? La migration des jeunes d'hier et d'aujourd'hui, Québec, Presses de l'Université Laval, pp. 105-130.

GRAFMEYER, Yves (1994) Sociologie urbaine, Paris, Éditions Nathan, 127 p.

HALARY, Charles (1994) Les exilés du savoir. Les migrations scientifiques internationales et leurs mobiles, Paris, L'Harmattan, $301 \mathrm{p}$.

HILY, Marie-Antoinette et Michel ORIOL (1993) Deuxième génération portugaise : la gestion des ressources identitaires, Revue européenne des migrations internationales, vol. 9, n 3, pp. 81-93.

HIRSCHHORN Monique et Michel BERTHELOT Éds. (1996) Mobilités et ancrages. Vers un nouveau mode de spatialisation, Paris, L'Harmattan, pp. 155-157. 
IREDALE, Robyn (2000) Migration policies for the highly skilled in the Asia-Pacific region, International Migration Review, vol. 34, no 3, pp. 882-906.

LATRECHE, Abdelkader (2001) Les migrations étudiantes de par le monde, Hommes \& Migrations, Hors dossier Nouvelles mobilités, $\mathrm{n}^{\circ}$ 1233, septembre-octobre, pp. 13-27.

LE GOFF, Jacques (1960) Les intellectuels au Moyen Âge, Paris, Éditions du Seuil, 191 p.

LÉVY, Jacques et Michel LUSSAULT (2003) Dictionnaire de la géographie, Paris, Belin, 1033 p.

MA MUNG, Emmanuel (1999) La dispersion comme ressource, in Jocelyne Cesari Éds., Les anonymes de la mondialisation, Paris, L'Harmattan, pp. 89-103.

MASSIT-FOLLÉA, Françoise et Françoise ÉPINETTE (1992) L'Europe des universités. L'enseignement supérieur en mutation, Paris, La Documentation française, $173 \mathrm{p}$.

MEINTEL, Deirdre (1993) Transnationalité et transethnicité chez des jeunes issus de milieux immigrés à Montréal, Revue européenne des migrations internationales, vol. 9, n³ 3, pp. 63-79.

NICOLE-DRANCOURT, Chantal et Laurence ROULLEAU-BERGER (2001) Les jeunes et le travail. 1950-2000, Paris, PUF, 266 p.

OCDE (2001) La mobilité des étudiants entre et vers les pays de l'OCDE : une analyse comparative, Tendances des migrations internationales SOPEMI, Système d'observation permanente des migrations, rapport annuel, pp. 100-126.

OCDE (2004a) Trends in international migration SOPEMI: main trends in international migration, vol. 2004, no 2, pp. 20-105.

OCDE (2004b) Indicators C3: Foreign Students in Tertiary Education, Education at a Glance 2004 Tables, http://www.oecd.org/dataoecd/28/54/33674158.xls, dernière consultation le 23 octobre 2005.

PICHON, Laure-Anne, Maurice COMTE et Xavier POULARD (2002) Les étudiants en séjour d'études à l'étranger. Qui ? Pourquoi ? Comment ?, Observatoire Universitaire Régional de l'Insertion Professionnelle (OURIP), Étude n 2002-02, 66 p.

PIERRE, Philippe (2003) Mobilité internationale et identités des cadres : des usages de l'ethnicité dans l'entreprise mondialisée, Fontenay-sous-bois (Val-de-Marne) : SIDES, 253 p.

RÉGION RHÔNE-ALPES (2004) Mobilité internationale. Statistiques BRFE/ SIME/ PEPSE, Charbonnièresles-Bains, Direction de l'enseignement supérieur.

RÉMY, Jean et Liliane VOYÉ (1992) La ville : vers une nouvelle définition ?, Paris, L'Harmattan, 174 p.

RICARD, Philippe (2001) Mobilité européenne ? Ça bouge... trop peu, Le Monde Campus, supplément au monde $\mathrm{n}^{\circ} 17$ 453, p. 34-36.

ROULLEAU-BERGER, Laurence (1991) La ville intervalle : jeunes entre centre et banlieue, Paris, Méridiens Klincksieck, 211 p.

ROULLEAU-BERGER, Laurence (1995) Expériences et compétences des jeunes dans les espaces intermédiaires, Lien social et politiques - RIAC, n³4, automne, pp. 109-117.

ROULLEAU-BERGER, Laurence (1999) Le travail en friche. Les mondes de la petite production urbaine, Saint-Étienne, Éditions de l'Aube, 245 p.

ROULLEAU-BERGER, Laurence et Lu SHI (2004) Routes migratoires et circulations en Chine : entre mobilités intracontinentales et transnationalisme, Revue européenne des migrations internationales, $20, \mathrm{n}^{\circ} 3$, pp. 7-27. 
SANTELLI, Emmanuelle (1999) Les enfants d'immigrés algériens et leur pays d'origine. Modes de relations économiques et professionnelles, Revue européenne des migrations internationales, vol. 15, $\mathrm{n}^{\circ} 2$, pp. 141-166.

SCHNAPPER, Dominique (1999) La compréhension sociologique. Démarche de l'analyse typologique, Paris, Presses universitaires de France, 125 p.

SIMON, Gildas (2001) Les nouvelles mobilités internationales, in Serge Lafitte (dossier réalisé par), « Un monde en migrations », ITA : les concours génériques à l'essai, Journal du CNRS, nº 134, février, pp. 12-14.

SIMON, Valérie (2000) La migration des étudiants maghrébins en France : une approche sociohistorique (1962-1994), in Vincent Geisser Éd., Diplômés maghrébins d'ici et d'ailleurs. Trajectoires sociales et itinéraires migratoires, Paris, CNRS Éditions, pp. 245-269.

SLAMA, Serge (1999) La fin de l'étudiant étranger, Paris, L'Harmattan, 312 p.

TARRIUS, Alain (1992) Les fourmis d'Europe. Migrants riches, migrants pauvres et nouvelles villes internationales, Paris, L'Harmattan, $207 \mathrm{p}$.

TARRIUS, Alain (2000) Les nouveaux cosmopolitismes. Mobilités, identités, territoires, La Tour d'Aigues, Éditions de l'Aube, $266 \mathrm{p}$.

UNESCO (2004) Recueil de données mondiales sur l'éducation 2004. Statistiques comparées sur l'éducation dans le monde, Institut de statistique de l'UNESCO, Montréal, 155 p.

VANISCOTTE, Francine, Aude HOUGUENAGUE et Anne WEST (2003) La mobilité étudiante en Europe, mythe ou réalité ? Comparaison France - Royaume-Uni, Paris, L'Harmattan, 248 p.

VERQUIN, Béatrice (2000) Du « modèle migratoire colonial » à la circulation des élites professionnelles. Le cas des Français à l'étranger, Thèse de doctorat de géographie, Université de Poitiers, 593 p.

WAGNER, Anne-Catherine (1998) Les nouvelles élites de la mondialisation : une immigration dorée en France, Paris, PUF, $236 \mathrm{p}$.

WEBER, Max (1965) Essais sur la théorie de la science, Paris, Plon, 537 p.

\section{NOTES}

1. Estimations rapportées par Coulon et Paivandi $(2003: 4)$ à partir des données de l'UNESCO et de l'OCDE.

2. Ces chiffres ne concernent que les mobilités réalisées dans le cadre du programme Erasmus et ne couvrent donc pas la totalité des Français qui étudient à l'étranger, lesquels sont estimés au nombre de 50593 en 2002 (OCDE, 2004b : C3.7). Pour les difficultés méthodologiques associées aux données disponibles sur les étudiants étrangers, se référer aux publications suivantes de l'OCDE (2001) et de l'UNESCO (2004).

3. Ce discours n'était pas exemptde paternalisme ni complètement désintéressé (Simon, 2000). On attendait que les futures élites des pays en voie de développement, formées à la « culture » et aux «valeurs» françaises, seraient les vecteurs du renforcement de la position de la France sur la scène internationale, du maintien d'une certaine influence politique dans les anciennes colonies (en contexte, faut-il le rappeler, de guerre froide), ainsi que de l'obtention de nouveaux marchés pour l'industrie française alors en pleine reconstruction.

4. Avec les poussées démographiques, le développement du système d'éducation primaire et secondaire des pays en voie de développement et l'insuffisance des structures d'enseignement supérieur à recevoir cette masse de nouveaux élèves, ces flux passent de la migration d'une élite 
issue des familles privilégiées et formée dans les lycées français à une migration de masse dont les acteurs sont de plus en plus souvent originaires des classes moyennes.

5. Nous pensons notamment ici aux travaux de Tarrius (1992), Wagner (1998), Findlay et al. (1999), Iredale (2000), Verquin (2000), Simon (2001) et Pierre (2003).

6. Ce travail a été mené dans une perspective comparative France/Québec et repose sur quatrevingt récits biographiques de jeunes ayant effectué au moins un séjour d'études à l'étranger dans le cadre de leur formation universitaire et sur plus d'une trentaine d'entretiens dirigés récoltés auprès d'acteurs institutionnels de la mobilité juvénile internationale. Les analyses présentées ici ne concernent que la partie française de l'enquête, soit 24 femmes et 16 hommes français âgés de 24 à 31 ans. Ces individus, issus de secteurs disciplinaires variés, étaient partis étudier pendant au moins une année scolaire (8-9 mois) dans un autre pays entre 1997 et 2000. Tous étaient originaires de la région lyonnaise et avaient obtenu une bourse de formation à l'étranger (BRFE) de la Région Rhône-Alpes (305 e/mois). Ils devaient être de retour en France et devaient se trouver en situation d'emploi ou de recherche d'emploi au moment de l'entretien, soit au printemps-été 2003. Une fois la récolte des récits terminée, il s'est avéré que la majeure partie des 40 enquêtés provenait des sciences humaines et sociales, des langues, des sciences de l'administration et des finances, et des sciences pour l'ingénieur. Presque tous ont réalisé leur séjour d'études au niveau de la licence et de la maîtrise, le plus souvent dans un autre pays européen ou dans la province de Québec. Au moment de l'entretien, 21 d'entre eux possédaient un diplôme de 2e cycle et 15 un diplôme de 3e cycle (DESS, DEA, Doctorat). Dix-neuf enquêtés sur 40 avaient un contrat à durée indéterminée (CDI), six un contrat à durée déterminée (CDD), quatre travaillaient en intérim et 11 étaient à la recherche d'un emploi (Garneau, 2006).

7. Extraits du rapport Dischamps cités par Borgogno et Streiff-Fenart (1996: 80) et Slama (1999: 93).

8. En mémoire de l'humaniste hollandais Érasme, intellectuel voyageur et fervent défenseur de la liberté de l'esprit. Le programme permet aux étudiants de niveau universitaire de se rendre dans un établissement européen avec lequel leur université ou leur école d'origine a signé un accord bilatéral de coopération. Des bourses Erasmus, d'une valeur d'environ 50 e par mois, peuvent également être octroyées aux participants.

9. Selon les responsables d'Erasmus en France, qui regrettent la faiblesse des budgets alloués par la Commission européenne à la mobilité des étudiants, la région Rhône-Alpes constitue «l'exception heureuse » (Ricard, $2001: 35$ ).

10. Il s'agit de la proportion avancée lors des XIVe Journées nationales Socrates-Leonardo de l'enseignement supérieur tenues à Lyon le 19 mai 2003.

11. Selon une enquête réalisée auprès d'étudiants de la Région Rhône-Alpes ayant effectué un séjour d'études à l'étranger en 1997-1998 dans le cadre d'un dispositif institutionnel, 35,5 \% des partants ont leur chef de famille dans la catégorie «cadres et professions intellectuelles supérieures » (contre 32,2 \% de l'ensemble des inscrits) et 21,7 \% dans la catégorie « employés et ouvriers " (contre 21,0 \% des inscrits) (Pichon, Comte et Poulard, 2002:17). Ces données laissent supposer que l'inégalité de l'accès à l'espace international agit moins au moment de partir - puisqu'à statut égal, la proportion de ceux qui partent est sensiblement la même que le nombre d'inscrits - que préalablement, lors de l'accès à l'enseignement supérieur. On peut penser que les supports institutionnels financiers apportés à la mobilité des étudiants dans cette région ont favorisé une certaine démocratisation des séjours d'études à l'étranger qui ne se retrouve cependant pas dans tout le pays.

12. Lévy et Lussault (2003: 124) définissent le capital spatial comme un "ensemble de ressources, accumulées par un acteur, lui permettant de tirer avantage, en fonction de sa stratégie, de l'usage de la dimension spatiale de la société ». Certaines dimensions de la réalité sociale d'une importance secondaire à un moment donné de l'histoire des collectivités peuvent devenir, avec la transformation des sociétés, des biens socialement valorisés susceptibles d'intervenir sur la 
hiérarchisation des groupes sociaux. Avec le développement des technologies de transport et de communication et la division internationale de la production, l'espace apparaît comme un bien social dont la mobilisation agira à son tour sur les représentations et les actions sociales (Rémy et Voyé, 1992 ; Grafmeyer, 1994 ; Hirschhorn et Berthelot, 1996 ; Ma Mung, 1999 ; Roulleau-Berger et Shi, 2004).

13. Les jeunes aux expériences multispatiales intégrées développent ce que nous sommes tentée de qualifier, à la suite des savoir-circuler dont parle Tarrius (2000), des « savoir-s'informer » qui s'expriment dans une grande capacité à naviguer sur le Web et à s'adresser à toute source d'informations susceptibles de les aider à définir et à organiser leur projet de partir. Ils ont une connaissance surprenante des organismes et institutions qui proposent des programmes de mobilité juvénile, des principaux sites Internet et des dates limites des concours.

14. D'autres pays signent également ces accords de réciprocité et opèrent une sélection, notamment sur une base financière, des candidats étrangers. Les pays anglo-saxons tels que les États-Unis, l'Angleterre et le Canada en sont un exemple, augmentant les droits de scolarité imputés aux non-nationaux.

15. Cette hiérarchisation ne manque certainement pas d'être rigidifiée par le classement académique des universités mondiales établi depuis 2003 par des chercheurs de l'Université Jiao Tong de Shanghai (ARWU, 2005).

16. "L'entrée dans la course et dans la concurrence pour le titre scolaire de fractions jusque-là faiblement utilisatrices de l'école a eu pour effet de contraindre les fractions de classe dont la reproduction étant assurée principalement ou exclusivement par l'école à intensifier leurs investissements pour maintenir la rareté relative de leurs titres et, corrélativement, leur position dans la structure des classes, le titre et le système scolaire qui le décerne devenant ainsi un des enjeux privilégiés d'une concurrence entre les classes qui engendre un accroissement général et continu de la demande d'éducation et une inflation des titres scolaires » (Bourdieu, $1978: 3$ ).

\section{RÉSUMÉS}

La globalisation de l'économie, les processus de construction régionale et l'évolution des politiques d'immigration participent conjointement à la transformation du paysage migratoire estudiantin. On assiste non seulement à une augmentation globale du nombre d'étudiants étrangers dans le monde, mais il semble également se dessiner une tendance à l'accroissement des circulations étudiantes Nord-Nord dans le cadre d'accords institutionnels fondés sur la réciprocité, à l'augmentation des dispositifs d'accueil des étudiants du Nord et au resserrement des politiques d'accueil de ceux en provenance du Sud. A partir d'une recherche menée auprès de quarante jeunes français au Québec, l'auteur questionne l'égalité d'accès des étudiants à l'espace international et, corollairement, aux postes qualifiés et aux secteurs compétitifs de la nouvelle économie. En recourant à l'analyse typologique, l'article montre comment les opportunités institutionnelles se joignent aux ressources personnelles et familiales initiales de l'étudiant pour produire une différenciation de la rentabilisation des expériences internationales sur les marchés $\mathrm{du}$ travail. La globalisation de l'économie, l'internationalisation de l'éducation et l'institutionnalisation grandissante de la mobilité étudiante internationale interagissent avec les stratégies d'insertion professionnelle et les représentations subjectives des étudiants pour engendrer une hiérarchisation des parcours migratoires et des socialisations professionnelles 
ainsi qu'une valorisation différenciée des filières, des établissements et des espaces nationaux d'enseignement supérieur à l'échelle internationale.

The Differentiation of French Students' Migratory Experiences. From International Student Mobility to the Circulation of Professional "Elites"? Economic globalization, regional construction processes and the evolution of immigration policies take part jointly in a transformation of the student migratory landscape. Not only is the worldwide number of international students increasing, but one can observe the multiplication of North-North student circulations made possible by institutional reciprocity agreements, while recognizing a tendency in the politics to restrict circulation of students from the South. Based upon a research study of 40 young French students targeted by recent programs for student mobility, the author discusses the equality of access by students to international space and consequently, to high skilled jobs in competitive sectors of the new economy. Using a typological analysis, the article shows how institutional opportunities and students' initial personal and familial resources combine to produce a differentiation of returns once on the job market. Economic globalization, internationalization of education and growing institutionalization of international student mobility interact with professional strategies and students' subjective representations to generate a hierarchy of migratory paths and professional socializations, resulting in different valorisations of educational fields, universities and graduate educational systems on an international scale.

Las experiencias migratorias diferenciadas de estudiantes franceses. ¿De las movilidades de estudiantes a la circulación de las élites profesionales? La globalización de la economía, los procesos de construcción regional y la evolución de las políticas de inmigración participan en la transformación del paisaje migratorio estudiantil. No sólo asistimos a un aumento global del numero de estudiantes extranjeros en el mundo sino que otra tendencia aparece: las circulaciones de estudiantes Norte-Norte crecen bajo los auspicios de acuerdos institucionales fundados en la reciprocidad y en el aumento de los dispositivos de acogida de estudiantes del Norte. Esta evolución contrasta con el endurecimiento de las políticas de acogida de estudiantes originarios del Sur. A partir de una investigación cuya muestra es un grupo de cuarenta jóvenes franceses de Québec, el autor cuestiona la igualdad de los estudiantes en el acceso al espacio internacional y, su corolario, a los puestos cualificados y a los sectores competitivos de la nueva economía. Recurriendo a un análisis por tipos, el artículo muestra cómo las oportunidades institucionales se aúnan a los recursos personales y familiares iniciales del estudiante para generar una diferenciación en la rentabilización de las experiencias internacionales en los mercados de trabajo. La globalización de la economía, la internacionalización de la educación y la institucionalización progresiva de la movilidad estudiantil internacional interaccionan con las estrategias de inserción profesional y las representaciones subjetivas de los estudiantes. Emergen, de este modo, una jerarquía de las rutas migratorias y de las socializaciones profesionales así como una valorización diferenciada de los sectores, de los establecimientos y de los espacios nacionales de formación superior a escala internacional.

\section{INDEX}

Mots-clés : étudiants, Français, mobilité géographique, socialisation, élite, insertion professionnelle 
AUTEUR

\section{STÉPHANIE GARNEAU}

Professeure adjointe, Université d'Ottawa, École de service social, 43, rue Templeton, K1N 6N5,

Canada. courriel : sgarneau@uottawa.ca 